\title{
Alumnado que no sigue las actividades educativas: El caso de una escuela de alta complejidad durante el confinamiento por COVID-19
}

\author{
Laura Fernández-Rodrigo ${ }^{1}$
}

\section{Introducción}

Los centros de alta complejidad se caracterizan por el bajo nivel socioeconómico de las familias de sus alumnos, por la concentración de alumnado con necesidades educativas específicas, por tener alumnos de distintas culturas e inmigrantes recién llegados (Departamento de Enseñanza, 2014). Las estadísticas indican que el 25\% del alumnado en estos centros obtiene un nivel bajo en las pruebas de competencias básicas, cuando el porcentaje se reduce al 6\% en los centros de baja complejidad. En la actualidad, nos encontramos con que un $17 \%$ de las escuelas de educación infantil y primaria en Cataluña son de alta complejidad (Riera, 2018).

Una de las medidas del estado de alarma a causa del COVID-19 fue el cierre de centros educativos desde el 13 de marzo de 2020. Los centros educativos se han movilizado para realizar la docencia de modo virtual siguiendo las indicaciones del Departamento de Educación (Departament d'Educació, 2020a). Sin embargo, cuando focalizamos esta situación en centros de alta complejidad, se dan una serie de problemas como los descritos en los siguientes apartados, elaborados a partir de aportaciones de la literatura y de la propia experiencia.

\subsection{Los niños y las niñas están más expuestos a factores de riesgo}

Los factores de riesgo se conocen como las evidencias, desventajas y elementos que causan estrés en la vida de las personas y que producen impactos negativos y deficiencias en su desarrollo evolutivo (Little, Axford, Morpeth, 2004). El hecho de que las escuelas estén cerradas provoca que el alumnado en situación de vulnerabilidad pase todo el tiempo en su entorno familiar, teniendo en primera línea que presenciar cualquier situación de inestabilidad económica y familiar, problemas de salud mental de familiares y violencia doméstica, por lo que su salud mental y física puede verse directamente afectada (Martínez de Lizarrondo, Etayo, Herrero, 2017; Molina, Vaquero, Vázquez, 2017).

\subsection{El Smartphone como único dispositivo para seguir la actividad escolar}

El Departamento de Educación en Cataluña promueve el uso de entornos virtuales creados con Moodle como herramienta para la docencia virtual. Además, ha puesto en marcha una iniciativa para proporcionar dispositivos móviles e Internet a familias en situación de vulnerabilidad para evitar la brecha digital (Departament d'Educació, 2020a). Sin embargo, los dispositivos son limitados y se da prioridad a los cursos de niveles educativos más altos. Por otro lado, las estadísticas demuestran que son muchas las familias que, pese a su baja situación socioeconómica, pueden disponer de un Smartphone con acceso a Internet, aunque sea el único dispositivo tecnológico en la unidad familiar (Consejo Económico y Social de España, 2017).

\subsection{Los padres y las madres carecen de competencias parentales y digitales}

Los padres y las madres en situación de vulnerabilidad suelen tener pocos recursos y habilidades para proporcionar atención básica, seguridad, establecer normas y límites y proporcionar una educación adecuada y libre de violencia a sus hijos e hijas (Balsells et al., 2015). Delante del confinamiento por COVID-19, los padres y las madres se pueden

\footnotetext{
Departament d'Educació de la Generalitat de Catalunya.

E-mail: 1ferna17@xtec.cat
} 
encontrar con dificultades para establecer una rutina y un ambiente confortable de estudio y un seguimiento de las tareas de sus hijos. Además, las familias en situación de vulnerabilidad carecen de competencias digitales (Suarez, Rodrigo, Muneton, 2016), por lo que pueden tener dificultades para poder ayudar a sus hijos a utilizar herramientas tecnológicas.

\subsection{Algunos maestros y maestras necesitan apoyo para la docencia virtual}

El Departamento de Educación de Cataluña y los grados de magisterio promueven la formación e iniciativas para mejorar las competencias digitales de los maestros y maestras. Sin embargo, encontramos que cerca de un $40 \%$ de los docentes en la educación pública se considera que tienen una competencia digital básica (Riera, 2018). Estos docentes tienen más dificultades que otros para proponer actividades utilizando estas herramientas y para gestionar entornos virtuales, especialmente los de mayor edad (Díaz, Cebrían, Fuster, 2016).

\section{Experiencia en una escuela de alta complejidad}

El estudio tiene como objetivo describir la experiencia de una escuela pública de alta complejidad de educación infantil y primaria en Cataluña frente el estado de alarma y el confinamiento por COVID-19. Se presentan las medidas adoptadas por el centro educativo así como las cifras del alumnado que sigue la actividad escolar desde sus hogares.

\subsection{Medidas tomadas durante el confinamiento}

El Departamento de Educación inició el período de docencia virtual a partir del 13 de abril de 2020 (Departament d'Educació, 2020b). A partir de esta fecha, el centro educativo tuvo en cuenta las directrices del departamento así como el contexto socioeconómico de las familias para adoptar las siguientes medidas:

a. Mantener el contacto con las familias a través de llamadas. Ante la imposibilidad de realizar videoconferencias con el todo el alumnado, el tutor o tutora del grupo realiza una llamada semanal a cada familia. La finalidad en estas llamadas va más allá de un seguimiento académico. A la vez, se les apoya emocionalmente, las familias se sienten acompañadas y tienen un recurso con el que contar en el caso de necesidad (Balsells, Vaquero, Ciurana, 2019).

b. Utilizar la web de la escuela, correo electrónico y WhatsApp. Se considera inviable incorporar un entorno virtual creado con Moodle cuando nunca se había hecho con anterioridad y cuando no se aseguran unas condiciones básicas e infraestructura tecnológica (Fernández-Rodrigo, Vaquero Tió, Balsells Bailón, 2019). Por lo tanto, se emplea como principal entorno virtual la web de la escuela. Para cada nivel educativo se comparte la lista de tareas semanales y una dirección de correo electrónico donde entregarlas a través de fotografías. El equipo directivo dispone del Smartphone de la escuela, en el que gestiona un grupo de WhatsApp por nivel educativo. Las familias preguntan sobre tareas, envían fotografías de las actividades y, al mismo tiempo, mantienen el contacto.

c. Proponer pocas actividades, sencillas y semanales. Cada maestro se responsabiliza de un área curricular y propone una actividad semanal. Las actividades son retos, propuestas artísticas, lecturas, breves escritos, ejercicios en páginas web educativas externas y ejercicios que pueden realizar en su libreta sino tienen la posibilidad de imprimir la ficha.

d. Considerar Google Forms. La herramienta libre de Google (Kalnow, et al., 2019) permite crear cuestionarios sencillos, es accesible para Smartphone y permite que los docentes reciban los resultados de cada alumno en una hoja de cálculo gestionada con Google Drive. La herramienta se utiliza para realizar actividades de comprensión a través de un vídeo o de una breve lectura con preguntas de verdadero o falso o de escoger la opción correcta. Del cuestionario elaborado, se obtiene el enlace y se comparte en la lista de tareas del nivel pertinente en la web de la escuela.

e. Proporcionar recursos no tecnológicos. En los casos en los que la familia no dispone de ningún tipo de dispositivo ni Internet, el equipo docente se ha movilizado para proporcionar un kit de material escolar y de un dosier de actividades.

f. Promover la cooperación entre el equipo docente. Los docentes se organizan a través de grupos de WhatsApp para compartir las tareas en la web de la escuela y para responder los correos electrónicos. Para apoyar a los docentes con menos conocimientos tecnológicos, aquellos que conocen más recursos realizan y comparten propuestas y videotutoriales explicativos.

\subsection{El alumnado que sigue la actividad escolar en cifras}

Los tutores han realizado un seguimiento de los alumnos que entregan por correo electrónico o WhatsApp las actividades propuestas en la web. Los resultados presentan el número y porcentaje de alumnos de educación primaria que entregan las actividades de forma regular frente al número total del alumnado por cada nivel educativo. 
Tabla 1. Número y porcentaje de alumnos de educación primaria que entregan actividades por nivel educativo.

\begin{tabular}{|l|c|c|c|}
\hline \multirow{2}{*}{ Nivel } & \multicolumn{2}{|c|}{ Alumnos que entregan actividades } & Total de alumnos \\
\cline { 2 - 4 } & $\mathbf{N}^{\mathbf{0}}$ & $\mathbf{\%}$ & $\mathbf{N}^{\mathbf{0}}$ \\
\hline $1^{\circ}$ & 5 & $19,23 \%$ & 26 \\
\hline$* 2^{\circ}$ & 20 & $38,46 \%$ & 52 \\
\hline$* 3^{\circ}$ & 27 & $60,00 \%$ & 45 \\
\hline$* 4^{\circ}$ & 23 & $50,00 \%$ & 46 \\
\hline$* 5^{\circ}$ & 11 & $23,91 \%$ & 46 \\
\hline$* 6^{\circ}$ & 17 & $39,53 \%$ & 43 \\
\hline Total & 103 & $39,92 \%$ & 258 \\
\hline
\end{tabular}

*Niveles de doble línea, en los que se han contabilizado el alumnado de las dos aulas.

Los resultados de la Tabla 1 demuestran que no se da ninguna relación entre los alumnos de niveles más bajos frente los de niveles más altos en referencia a las tareas entregadas. El alumnado que entrega más actividades es el de $3^{\circ}(60 \%), 4^{\circ}(50 \%)$ y $6^{\circ}(39,53 \%)$. Por otro lado, el alumnado que entrega menos actividades es el de $1^{\circ}(19,23 \%)$, $5^{\circ}(23,91 \%)$ y $2^{\circ}(38,46 \%)$. El alumnado de cursos superiores se considera más autónomo y con más conocimientos tecnológicos y digitales para poder seguir las actividades virtuales. Sin embargo, el apoyo recibido de los padres y las madres puede que sea el factor que favorezca a los alumnos de menor edad para realizar las actividades.

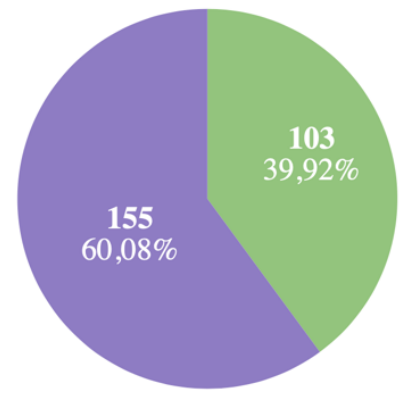

- Alumnado que sí entrega las actividades

Alumnado que no entrega las actividades

Figura 1. Porcentaje del alumnado de educación primaria que entrega las actividades.

En toda la etapa de educación primaria (Figura 1), solo un 39,92\% del alumnado realiza las actividades escolares propuestas a través de la página web de la escuela. Asimismo, se demuestra que más de la mitad del alumnado de educación primaria $(60,08 \%)$ no entrega las actividades escolares. Entre estos, encontramos tres tipos de casos: a) alumnado con el que no se ha podido contactar por ningún medio; b) 10 alumnos que no tienen medios tecnológicos y a los que se les ha podido facilitar en sus hogares un kit de material escolar y un dosier de actividades; c) alumnado con el que se mantiene el contacto pero no entrega las actividades.

\section{Conclusiones}

Los resultados del estudio muestran que menos de la mitad del alumnado de educación primaria en un centro de alta complejidad entrega las actividades escolares. Este dato, sin tener en cuenta la evaluación de las actividades, pone de manifiesto cómo el confinamiento por COVID-19 está agravando el rendimiento académico de los niños y niñas en situación de vulnerabilidad.

La literatura demuestra cómo disminuir los factores de riesgo y promover una buena salud mental repercute directamente en una mejora del rendimiento académico del alumnado (Hart et al., 2018; Maitland, 2018). En este sentido, conviene establecer medidas con la finalidad de favorecer la igualdad educativa (Haretche, 2013).

Las medidas a corto plazo durante el confinamiento tendrían que poner el foco en elaborar un protocolo especial para los centros de alta complejidad, ya que significan un factor de protección para su alumnado (González-Arratia et al., Morelato, González, Ruiz, 2018). El protocolo tendría que considerar recursos humanos, económicos y tecnológicos para aumentar la comunicación entre las familias y el centro, y establecer medidas de seguridad para agilizar la vuelta a la escuela de los alumnos.

A largo plazo, se propone establecer iniciativas para toda la comunidad educativa, como promover la formación de docentes en competencias digitales y en infancia en situación de vulnerabilidad (Haretche, 2013; Riera, 2018) y 
la realización de programas socioeducativos de competencias parentales para las familias (Amorós-Martí et al.,2016; Balsells et al., 2015; Torres et al., 2015).

\section{Referencias bibliográficas}

Amorós-Martí, P., Byrne, S., Mateos-Inchaurrondo, A., Vaquero-Tió, E., Mundet-Bolós, A. (2016). "Learning together, growing with family": The implementation and evaluation of a family support programme. Psychosocial Intervention, 25(2), 87-93. https://doi.org/10.1016/j.psi.2016.02.002

Balsells, M. À., Pastor, C., Amorós, P., Fuentes-Peláez, N., Molina, M. C., Mateos, A., ... Ponce, C. (2015). Caminar en familia: Programa de competencias parentales durante el acogimiento y la reunificación familiar. Madrid: Ministerio de Sanidad Servicios Sociales e Igualdad.

Balsells, M. À., Pastor, C., Mateos, A., Vaquero, E., Urrea, A. (2015). Exploring the needs of parents for achieving reunification: The views of foster children, birth family and social workers in Spain. Children and Youth Services Review, 48, $159-166$. https://doi.org/10.1016/j.childyouth.2014.12.016

Balsells, M. À., Vaquero, E., Ciurana, A. (2019). El apoyo social durante el acogimiento: su relevancia para el bienestar de los niños y las niñas en situación de protección. Sociedad e Infancias, 3, 115-132. https://doi.org/10.5209/soci.63403

Consejo económico y social de España. (2017). Nuevos hábitos de consumo, cambios sociales y tecnológicos (en línea).

http://www.ces.es/documents/10180/3557409/Inf0416.pdf

Departament d'Educació. (2020a). Educació crea 615 entorns virtuals d'aprenentatge Moodle perquè tots els centres puguin dur a terme la docència en línia (en línea). https://govern.cat/salapremsa/notes-premsa/384084/educacio-crea-615-entorns-virtuals-aprenentatge-moodle-tots-centres-puguin-dur-terme-docencia-linia, acceso 2 de mayo de 2020.

Departament d'Educació. (2020b). Educació dona criteris a les direccions dels centres per dur a terme l'avaluació i el seguiment de l'alumnat durant el confinament (en línea). http://ensenyament.gencat.cat/ca/inici/nota-premsa/?id=384278, acceso 2 de mayo de 2020.

Departamento de Enseñanza. (2014). RESOLUCIÓN ENS/906/2014. Diari Oficial de La Generalitat de Catalunya, (661330.4.2014), $1 / 4$.

Díaz, I., Cebrían, S., Fuster, I. (2016). Las competencias en TIC de estudiantes universitarios del ámbito de la educación y su relación con las estrategias de aprendizaje. Revista ELectrónica de Investigación y EValuación Educativa, 22(1), 1-24. https:// doi.org/10.7203/relieve.21.1.8159

Fernández-Rodrigo, L., Vaquero Tió, E., Balsells Bailón, M. À. (2019). La percepción de los profesionales sobre el uso de un entorno virtual como medio de soporte en el programa socioeducativo 'Caminar en Familia.' Pixel-Bit, Revista de Medios y Educación, (56), 129-148. https://doi.org/10.12795/pixelbit.2019.i56.07

González-Arratia, N. I., Morelato, G., González, S., Ruiz, A. O. (2018). Factores de riesgo y protección para la resiliencia en niños mexicanos y argentinos. Aportaciones actuales de la psicología social (Vol. 4, p. 23).

Haretche, C. (2013). Mézclate conmigo. Boletín Del Instituto Nacional de Evaluación Educativa (en línea). https://www.ineed. edu.uy/sites/default/files/textosegregación sociocultural.pdf

Hart, A., Fernández-Rodrigo, L., Molina, M. C., Izquierdo, R., Maitland, J. (2018). L'Enfocament de Resiliència Acadèmica en la promoció de la salut mental dels adolescents. Proposta per a la seva aplicació en centres educatius. Lleida: Càtedra Educació i Adolescència Abel Martínez Oliva. Edicions de la Universitat de Lleida (en línea). https://repositori.udl.cat/handle/10459.1/64643

Kalnow, A., Lloyd, C., Casey, J., Little, A. (2019). Google Forms - A Novel Solution to Blended Learning. Journal of Education and Teaching in Emergency Medicine, 4(2). https://doi.org/https://doi.org/10.21980/J8BP77

Little, M., Axford, N., Morpeth, L. (2004). Research Review: Risk and protection in the context of services for children in need. Child \& Family Social Work, 9(1), 105-117. https://doi.org/10.1111/j.1365-2206.2004.00296.x

Maitland, J. (2018). School Staff Experiences of an Academic Resilience Approach: a Whole Systems Perspective. University of Brighton.

Martínez de Lizarrondo, A., Etayo, O., Herrero, I. (2017). Pobreza anclada y transmisión intergeneracional de la pobreza en Navarra. Sociedad e Infancias, 1, 239-259.https://doi.org/10.5209/SOCI.55913

Molina, M. C., Vaquero, E., Vázquez, N. (2017). La promoció de la salut mental dels adolescents en els centres educatius : resiliència i recursos digitals. Càtedra Educació i Adolescència, 2 (en línea).

https://repositori.udl.cat/bitstream/handle/10459.1/59705/Molina\%26etal_2017.pdf?sequence=1\&isAllowed=y

Riera, J. (2018). Reptes de l'educació a Catalunya. Anuari 2018. Barcelona (en línea). //www.fbofill.cat/sites/default/files/Anuari2018_190619.pdf

Suarez, A., Rodrigo, M. J., Muneton, M. (2016). Parental activities seeking online parenting support: Is there a digital skill divide? Revista de Cercetare Si Interventie Sociala, 54(September), 36-54.

Torres, A., Suárez, A., Álvarez, M., Padilla, S., Rodríguez, E., Rodrigo, M. J. (2015). Apoyo Parental Online. En M. J. Rodrigo, M. L. Máiquez, J. C. Martín, S. Byrne, B. Rodríguez (Eds.), Manual práctico en parentalidad positiva (pp. 245-263). Madrid: Síntesis. 